\title{
Applications of Holography to Strongly Coupled Plasmas
}

\author{
Carlo Ewerz, Konrad Schade* \\ Institut für Theoretische Physik, Universität Heidelberg, \\ Philosophenweg 16, 69120 Heidelberg, Germany \\ and \\ ExtreMe Matter Institute EMMI, GSI Helmholtzzentrum für Schwerionenforschung, \\ Planckstraße 1, 64291 Darmstadt, Germany \\ E-mail: c.ewerz@thphys.uni-heidelberg.de, \\ k. schadedthphys.uni-heidelberg.de
}

\begin{abstract}
We study several observables related to heavy quarks in strongly coupled plasmas using the gauge/gravity correspondence. Besides the $\mathrm{AdS}_{5}$ space dual to $\mathscr{N}=4$ supersymmetric YangMills theory we consider large classes of theories obtained from various deformations of the $\mathrm{AdS}_{5}$ space. Among them are theories that solve equations of motion of a 5-dimensional EinsteinHilbert-scalar action. Specifically, we calculate the screening distance of a heavy quark-antiquark pair moving at constant velocity through the plasma, the running coupling defined via the free energy of such a static pair, and the energy radiation from a heavy quark forced into a circular motion in the plasma. We find that these observables show universal behaviour in large classes of theories. The screening distance in these classes of theories, that is the maximal distance for which a heavy quark-antiquark pair is bound, is found to be bounded from below by its value in $\mathscr{N}=4$ super Yang-Mills theory.
\end{abstract}

Xth Quark Confinement and the Hadron Spectrum,

October 8-12, 2012

TUM Campus Garching, Munich, Germany

${ }^{*}$ Speaker. 


\section{Introduction}

Since its discovery the gauge/gravity (or AdS/CFT) correspondence $[1,2,3]$ has been used to explore various aspects of supersymmetric gauge theories at strong coupling, including their behavior at finite temperature. More recently, it was realized that this correspondence or duality has the potential to help answer questions also in the realm of various strongly coupled systems that are accessible to experiment. It turned out that a particularly interesting and fruitful application of the AdS/CFT correspondence is to the quark-gluon plasma created and studied at RHIC and LHC. These experiments find strong evidence that the quark-gluon plasma is in fact strongly coupled. New theoretical methods like the AdS/CFT correspondence are especially welcome here since the strong coupling makes ab initio calculations based on QCD very hard, leaving only lattice QCD as a reliable tool. Lattice QCD, however, can only address static properties reliably while it has difficulties in the calculation of many dynamical problems that are of interest in the study of the quark-gluon plasma. The AdS/CFT correspondence can address not only dynamical as well as static observables but in addition has the advantage that its results are obtained from computationally simple calculations on the gravity side.

In its original form, the AdS/CFT correspondence relates 4-dimensional $\mathscr{N}=4$ supersymmetric Yang-Mills theory (SYM) with gauge group $\mathrm{SU}\left(N_{c}\right)$ at strong coupling and taken in the large- $N_{c}$ limit to weakly coupled (super)gravity on a 5-dimensional Anti-de Sitter space $\mathrm{AdS}_{5}$. The 4-dimensional theory can be thought of as living 'on the boundary' of the AdS space, so that the equivalence of these two descriptions of the same underlying physics can actually be understood as a realization of the holographic principle. Finite temperature on the gauge theory side is implemented in the correspondence by putting a black hole in the bulk of the $\mathrm{AdS}_{5}$ space, such that the temperature of the gauge theory coincides with the Hawking temperature of the black hole.

But QCD and $\mathscr{N}=4$ SYM are two very different theories. A successful application of the correspondence to real-world QCD therefore appears far from obvious. At a finite temperature above the deconfinement temperature $T_{c}$ the situation is already more promising. On the one hand, chiral symmetry and confinement are lost in QCD. On the other hand, the supersymmetry and the conformal symmetry of $\mathscr{N}=4 \mathrm{SYM}$ are broken by finite temperature. One can therefore hope that QCD and SYM share at least some properties at finite temperature.

Considerable efforts have been made to find a holographic description of theories that are even closer to QCD than $\mathscr{N}=4 \mathrm{SYM}$. This can be achieved by considering deformations of the AdS space which can be introduced in various ways. A common feature of these deformations is that they in some way or another break the conformal invariance of $\mathscr{N}=4$ SYM explicitly. One line of thought is to construct theories that reproduce as many properties of QCD as possible, although an exact holographic dual of QCD would certainly be extremely difficult to find, and in fact not even known to exist. In a second line of thought - the one which we will follow here - one considers large classes of deformations and tries to identify universal properties of observables in these classes of holographic theories. An example of this approach is the well-known finding that the ratio of shear viscosity to entropy density $\eta / s$ equals $1 /(4 \pi)$ in large classes of theories $[4,5]$. Other observables might change only very little or might consistently change in a certain direction under the influence of deformations of the AdS space. If such a universal property is found one can hope that the behavior of QCD is not too different from the trend observed in these large classes of 
theories. In this talk we would like to present some interesting results obtained in this approach. A more detailed description is given elsewhere [6].

\section{AdS-type Gravity Models for Strongly Coupled Plasmas at Finite Temperature}

The gravity dual of $\mathscr{N}=4 \mathrm{SYM}$ at finite temperature is $\mathrm{AdS}_{5}$ with a black hole at a position $z_{h}$ in the 5th dimension,

$$
\mathrm{d} s^{2}=\frac{L_{\mathrm{AdS}}^{2}}{z^{2}}\left[-f(z) \mathrm{d} t^{2}+\mathrm{d} \vec{x}^{2}+\frac{\mathrm{d} z^{2}}{f(z)}\right]
$$

with

$$
f(z)=1-\frac{z^{4}}{z_{h}^{4}}
$$

The AdS curvature radius $L_{\mathrm{AdS}}$ is assumed to be large, corresponding to a large 't Hooft coupling of the gauge theory. The temperature of the dual $\mathscr{N}=4$ gauge theory is $T=1 /\left(\pi z_{h}\right)$.

In order to construct theories closer to real-world QCD one can consider various modifications of this metric. We will consider classes of theories with metrics of the general form

$$
\mathrm{d} s^{2}=\mathrm{e}^{2 A(z)}\left(-h(z) \mathrm{d} t^{2}+\mathrm{d} \vec{x}^{2}\right)+\mathrm{e}^{2 B(z)} \frac{\mathrm{d} z^{2}}{h(z)} .
$$

The corresponding temperature of the dual 4-dimensional theory is

$$
T=e^{A\left(z_{h}\right)-B\left(z_{h}\right)} \frac{\left|h^{\prime}\left(z_{h}\right)\right|}{4 \pi},
$$

where $z_{h}$ is again the position of the black hole horizon in the bulk of $\mathrm{AdS}_{5}, h\left(z_{h}\right)=0$. The $\mathscr{N}=4$ case (2.1) is recovered for $A(z)=B(z)=0$ and $h(z)=f(z)$.

A simple class of deformations of the $\mathscr{N}=4$ case was proposed in [7] and [8] motivated by the widely discussed soft-wall model at zero temperature [9]. This one-parameter class of metrics is obtained by either starting with a $T=0$ soft-wall model and inserting the black-hole factor (2.2) in the same way as in (2.1). Alternatively, one can with the same result start from (2.1) and multiply it by a soft-wall factor. We therefore call this the finite temperature soft wall model, $\mathrm{SW}_{T}$. Its metric (in the string frame, see below) has the form

$$
\mathrm{d} s^{2}=\frac{L_{\mathrm{AdS}}^{2}}{z^{2}} \mathrm{e}^{\frac{29}{20} c z^{2}}\left(-f(z) \mathrm{d} t^{2}+\mathrm{d} \vec{x}^{2}+\frac{\mathrm{d} z^{2}}{f(z)}\right) .
$$

The dimensionful parameter $c$ determines the non-conformality of the $\mathrm{SW}_{T}$ model. According to (2.4) the temperature of the $\mathrm{SW}_{T}$ model is again $T=1 /\left(\pi z_{h}\right)$. The main drawback of the $\mathrm{SW}_{T}$ model is that it is not a solution of a 5-dimensional gravitational action. Therefore its consistency as a theory is somewhat doubtful.

A class of consistent deformations of the $\mathrm{AdS}_{5}$ metric (2.1) can be constructed as solutions of a 5-dimensional Einstein-Hilbert-scalar action. We follow [10, 11] and start with the action

$$
S_{\mathrm{EHs}}=\frac{1}{16 \pi G_{\mathrm{N}}^{(5)}} \int \mathrm{d}^{5} x \sqrt{-G}\left(\mathscr{R}-\frac{1}{2}(\partial \Phi)^{2}-V(\Phi)\right),
$$


where $G_{N}^{(5)}$ is the 5-dimensional Newton constant, $\mathscr{R}$ is the Ricci scalar, and $V(\Phi)$ a potential for the scalar field $\Phi(z)$. $\Phi(z)$ can but need not be the dilaton. We consider both possibilities as independent models which have been termed 'string frame' and 'Einstein frame' model, respectively [11]. In order to obtain metrics that resemble the soft-wall type models one can consider classes of 2-parameter models of the form (2.3) by making the following ansatz. We switch to a gauge where the bulk coordinate is identified with the scalar $\Phi$ such that our general metric is

$$
\mathrm{d} s^{2}=\mathrm{e}^{2 A(\Phi)}\left(-h(\Phi) \mathrm{d} t^{2}+\mathrm{d} \vec{x}^{2}\right)+\mathrm{e}^{2 B(\Phi)} \frac{\mathrm{d} \Phi^{2}}{h(\Phi)} .
$$

We then choose $A(\Phi)$ and the scalar $\Phi(z)$ as

$$
A(\Phi)=\frac{1}{2} \log \left(\frac{L_{\text {AdS }}^{2}}{z^{2}}\right)-\frac{1}{2} c z^{2}, \quad \Phi=\sqrt{\frac{3}{2}} \phi z^{2}
$$

and determine $B(z)$, the horizon function $h(z)$, and the potential $V(\Phi)$ from the equations of motion of the action (2.6). The resulting class of metrics has $c$ and $\alpha \equiv c / \phi$ as two independent parameters characterizing the deformation [11]. The temperature is given by a formula analogous to (2.4) involving the horizon position. Note that we do not fix the scalar potential $V(\Phi)$ from the beginning but use the freedom to adjust it such that the solution has the desired form (2.8). The class of 2-parameter metrics obtained in this way defines consistent 4-dimensional theories via the holographic duality, but we should point out that in general we do not know their Lagrangian.

\section{Screening Distance and Running Coupling}

We first consider the screening of a heavy quark-antiquark pair moving with velocity $v$ in a plasma. This problem was first addressed for $\mathscr{N}=4 \mathrm{SYM}$ in [12] and for the $\mathrm{SW}_{T}$ model in [13]. The free energy $F(L)$ of such a system is given by the thermal expectation value of a timelike Wegner-Wilson loop, $\langle W(\mathscr{C})\rangle=\exp [-i \mathscr{T} F(L)]$ where $L$ is separation between the quark and antiquark, and $\mathscr{T}$ the large temporal extension of the loop. In the gravity dual, this situation is described by an open string connecting the quark and antiquark and hanging down into the bulk. For infinitely heavy quarks the ends of the string are at $z=0$. The expectation value can then be obtained as

$$
\langle W(\mathscr{C})\rangle \propto e^{i\left(S-S_{0}\right)},
$$

where $S$ is the Nambu-Goto action for the string worldsheet parametrized by $\sigma$ and $\tau$,

$$
S_{\mathrm{NG}}=-\frac{1}{2 \pi \alpha^{\prime}} \int \mathrm{d} \sigma \mathrm{d} \tau \sqrt{-\operatorname{det} g_{a b}},
$$

Here $g_{a b}=G_{\alpha \beta} \partial_{a} X^{\alpha} \partial_{b} X^{\beta}$ is the induced metric and $\alpha^{\prime}$ is the string slope. Further, $S_{0}$ is twice the action of a single quark, given by the Nambu-Goto action of an open string hanging down into the bulk. The calculation of the actions $S$ and $S_{0}$ has to be performed in the string frame. For the 2-parameter model that means that the Einstein frame metric discussed above is multiplied by an exponential of the dilaton, $G_{\alpha \beta}^{s}=\exp (-\sqrt{2 / 3} \Phi) G_{\alpha \beta}^{E}$ in our normalization of the dilaton. If the scalar $\Phi$ in the action (2.6) is not a dilaton, the string frame and the Einstein frame metrics coincide. 
For a given distance $L$ between the quark and antiquark up to a maximally possible distance $L_{\max }$ there are always two string solutions. They can be parametrized by the maximal depth $z_{c}$ that they reach in the bulk. For the simple case that the pair is not moving with respect to the plasma, the relation between $L$ and $z_{c}$ is given by

$$
L \pi T=2 \pi T \int_{0}^{z_{c}} \sqrt{\frac{h\left(z_{c}\right) e^{2 B(z)+4 A\left(z_{c}\right)-2 A(z)}}{h(z)^{2} e^{4 A(z)}-h(z) h\left(z_{c}\right) e^{4 A\left(z_{c}\right)}}} \mathrm{d} z,
$$

and a more complicated relation applies for $v \neq 0$. The solution with the larger $z_{c}$, that is the one coming closer to the horizon, turns out to have the higher energy and is hence the unstable solution. At distances larger than $L_{\max }$ there are no real-valued string solutions connecting the quark and antiquark. We call this maximally possible distance the screening distance. It should not be confused with the Debye screening length which characterizes the exponential flattening of the free energy at distances much larger than $L_{\max }$ where the quark and antiquark are no longer bound. (Also the Debye screening length can be addressed in the holographic setup, see [14].)

We have calculated the screening distance $L_{\max }$ in all models discussed above for all its possible kinematical parameters, namely the velocity $v=\tanh \eta$ of the pair in the plasma and its orientation angle $\theta$ with respect to the velocity. Figure 1 shows the dependence of $L_{\max }$ on the rapidity $\eta$ for the case $\theta=0$ for various models with typical values for the deformation parameters. We have chosen to plot the dimensionless quantity $\pi T L_{\max }$ for convenience and have divided out

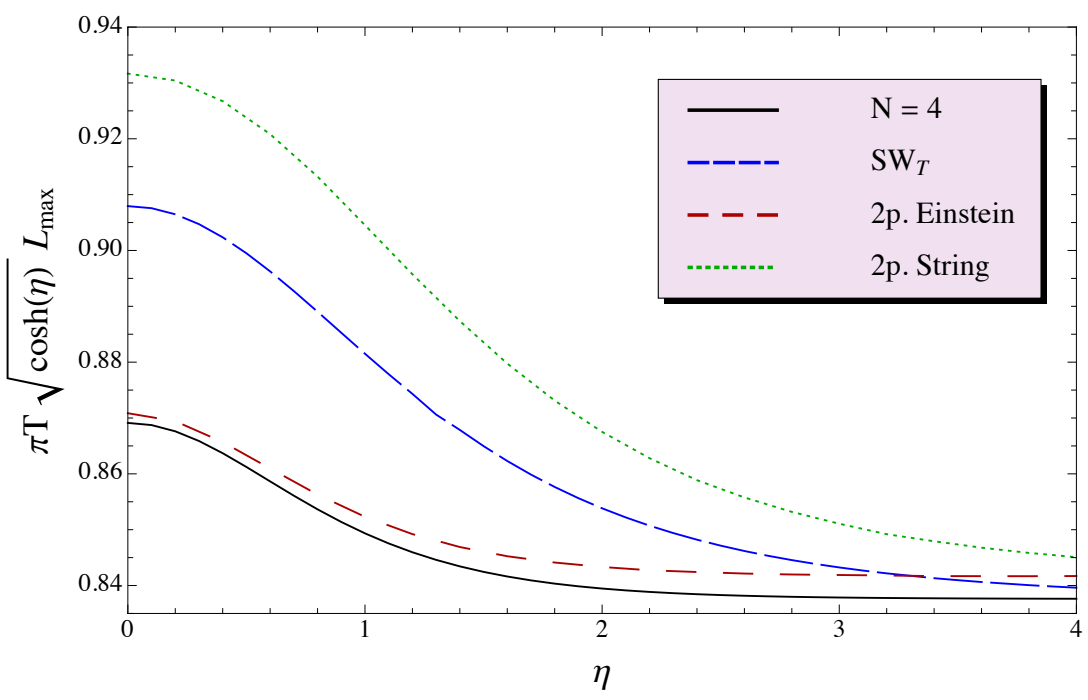

Figure 1: Dimensionless screening distance $\pi T L_{\max } \sqrt{\cosh \eta}$ of the $Q \bar{Q}$ pair in the plasma as a function of rapidity $\eta$.

the dominant factor $1 / \sqrt{\cosh \eta}$ found in the $\eta$-dependence.

We have made the interesting observation that in all deformations in the above classes of models and for all kinematical parameters the screening distance $L_{\max }$ turns out to be larger than the corresponding value in $\mathscr{N}=4 \mathrm{SYM}$. This leads us to conjecture that the screening distance in $\mathscr{N}=4 \mathrm{SYM}$ is a lower bound for all theories described by gravity duals. A general proof of this conjecture appears difficult. However, we have performed an analytic study of the problem for 
small perturbations of the metric around pure $\mathrm{AdS}_{5}$ space. It indicates that the conjecture holds in first order in the deformation of the metric under very mild and general assumptions [6].

The free energy $F(L)$ of the quark-antiquark pair can be extracted from (3.1) by computing $S$ and $S_{0} . S_{0}$ has been calculated in $\mathscr{N}=4 \mathrm{SYM}$ in $[15,16]$ and for the $\mathrm{SW}_{T}$ model in [17]. The extension to the models discussed here is straightforward. Once the free energy is known, one can proceed and calculate the running coupling in the whole class of models. It can be defined in terms of the free energy as

$$
\alpha_{q q}(L, T) \equiv \frac{3}{4} L^{2} \frac{\mathrm{d} F(L, T)}{\mathrm{d} L},
$$

where the normalization is motivated by the Casimir factor of QCD. In our setup the Lagrangian and hence the Casimir factor of the dual theory is not known, however. The normalization of the running coupling is further related to the 't Hooft coupling of the theory. As a consequence we treat the normalization of the result in our models as a free parameter to be suitably fixed. In figure 2 we show as an example the running coupling in the $\mathrm{SW}_{T}$ model for various temperatures. We have chosen $T_{c}=176 \mathrm{MeV}$ for definiteness. The endpoint of the curves is always given by the screening

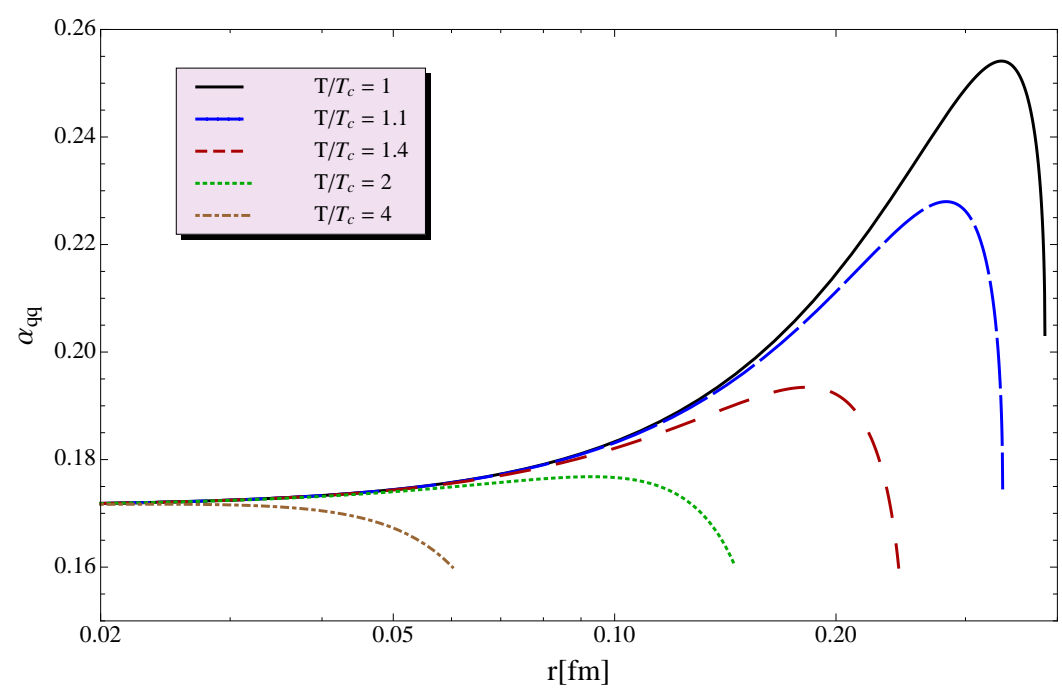

Figure 2: Running coupling $\alpha_{q q}$ versus the $Q \bar{Q}$-distance for several values of the temperature $T / T_{c}, T / T_{c}=$ $1,1.1,1.4,2$ and 4 and for fixed deformation $c / T^{2}, c / T^{2}=4$ in the $\mathrm{SW}_{T}$ model.

distance $L_{\max }$. It is a universal property of the running coupling $\alpha_{q q}$ in all non-conformal models that it develops a maximum before falling rather steeply close to $L_{\max }$. We further find that in the 2-parameter model it is possible to adjust the model parameters such that the running coupling quite closely resembles the lattice data for QCD found in [18].

\section{Energy Loss of a Rotating Quark}

The energy loss of an accelerated quark in a medium can give valuable information about the properties of the medium itself. We therefore consider the motion of a quark on a circle of radius $R_{0}$ at constant angular velocity $\omega$. This is certainly not a realistic situation in any experiment, but serves well as the simplest model for an accelerated motion to be treated in the gauge/gravity 
correspondence. The problem had been addressed so far only in $\mathscr{N}=4$ SYM: at zero temperature in [19], and at finite temperature in [20]. We have calculated the motion of the string attached to the rotating quark also in the deformed metrics discussed above [6].

In figure 3 some typical configurations are shown. The left panel shows the pure $\mathrm{AdS}_{5}$ case at $T=0$ for two different angular velocities. In the $T=0$ case there is no horizon and the string spirals out to infinite radii deep in the bulk. This situation changes at finite temperature due to the
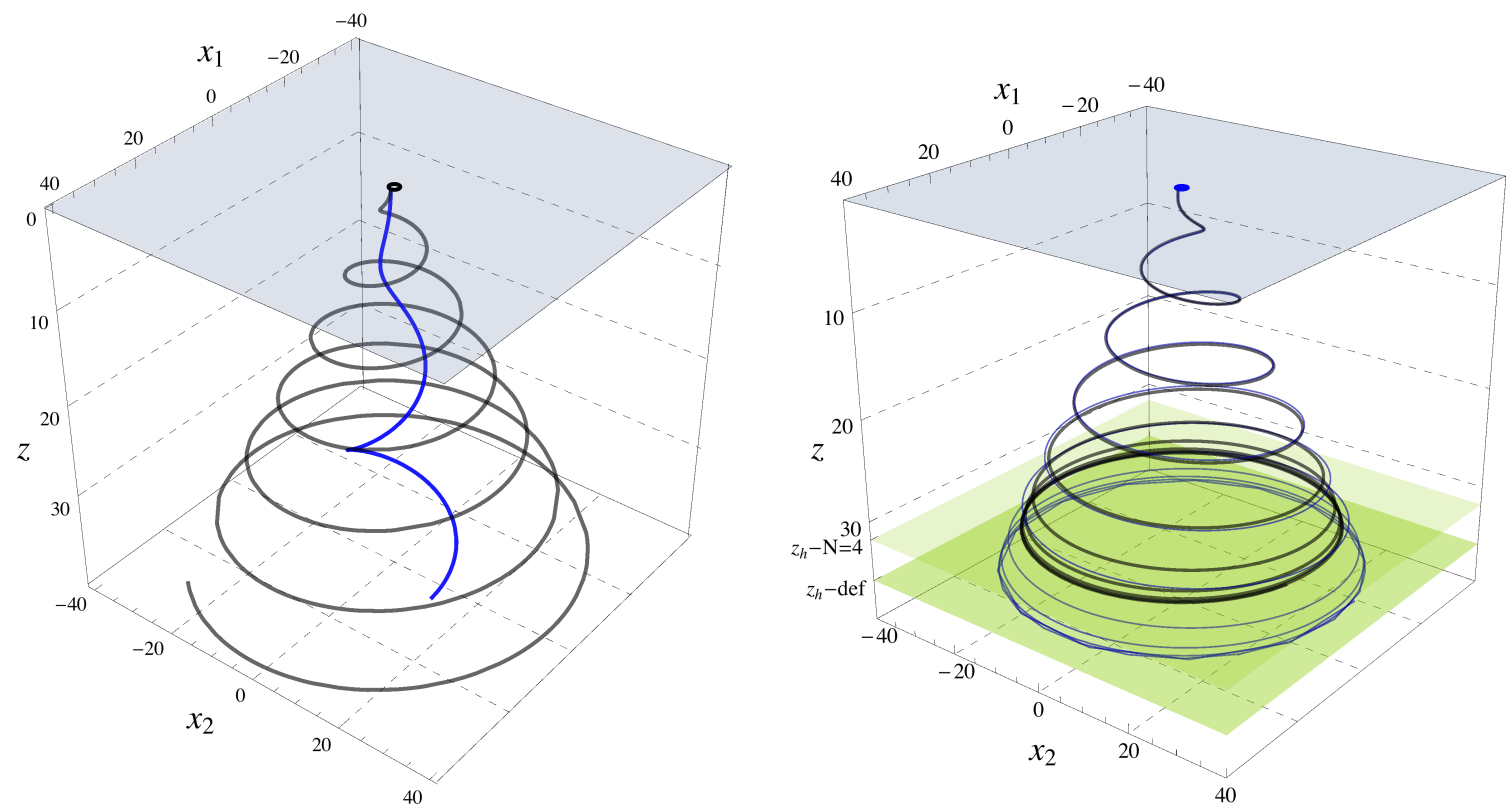

Figure 3: Left: String configuration in pure $\operatorname{AdS}_{5}\left(\mathscr{N}=4 \mathrm{SYM}\right.$ at zero temperature) and radius $R_{0}=1$ and angular velocity $\omega=0.3$ (blue) and 0.7 (black). The small black circle at the top represents the trajectory of the rotating quark. Right: String configuration in 2-parameter string-frame model (blue line) for typical values of $\alpha$ and $c$ together with the corresponding $\mathscr{N}=4$ case (black line) at $T=0.01, R_{0}=1$ and $\omega=0.7$ (in suitable dimensionless units).

horizon. Here the string extends only to a finite radius, see right panel.

For the deformed models we in general observe that the string solution deviates considerably from the one corresponding to $\mathscr{N}=4$ SYM only at larger depths in the bulk. A more detailed analysis further shows that the energy radiated from the quark is mainly determined by the uppermost part of string configuration. As a consequence one finds that the radiation pattern in the deformed theories is in general very close to the one in $\mathscr{N}=4 \mathrm{SYM}$.

\section{Summary}

We have used holographic methods to study various observables related to the motion of heavy quarks in the plasma of a variety of theories at finite temperature. We have in particular considered the screening distance, that is the maximal distance for which a heavy quark and antiquark form a bound state, for all velocities and orientation angles of the pair in the plasma. The value of the screening distance in $\mathscr{N}=4 \mathrm{SYM}$ is a lower bound for a large class of theories for all kinematical parameters. We conjecture this to hold for possibly all theories and find confirmation for this 
conjecture in a perturbative analysis. We have computed the running coupling from the free energy of a static quark-antiquark pair in the medium and find that it exhibits some universal features for large classes of models. Finally, we have considered a rotating quark in the plasma. Its energy radiation is very similar to the case of $\mathscr{N}=4 \mathrm{SYM}$ for all models that we study.

\section{Acknowledgments}

The work of K. S. was supported in part by the International Max Planck Research School for Precision Tests of Fundamental Symmetries. This work was supported by the Alliance Program of the Helmholtz Association (HA216/EMMI).

\section{References}

[1] J. M. Maldacena, Adv. Theor. Math. Phys. 2, (1998) 231 [Int. J. Theor. Phys. 38, (1999) 1113] [arXiv:hep-th/9711200].

[2] S. S. Gubser, I. R. Klebanov and A. M. Polyakov, Phys. Lett. B 428 (1998) 105 [arXiv:hep-th/9802109].

[3] E. Witten, Adv. Theor. Math. Phys. 2, (1998) 253 [arXiv:hep-th/9802150].

[4] G. Policastro, D. T. Son and A. O. Starinets, Phys. Rev. Lett. 87 (2001) 081601 [arXiv:hep-th/0104066].

[5] P. Kovtun, D. T. Son and A. O. Starinets, Phys. Rev. Lett. 94 (2005) 111601 [arXiv:hep-th/0405231].

[6] K. Schade, PhD thesis, Heidelberg University, 2012; C. Ewerz, K. Schade, in preparation

[7] O. Andreev and V. I. Zakharov, Phys. Rev. D 74 (2006) 025023 [hep-ph/0604204].

[8] K. Kajantie, T. Tahkokallio and J. T. Yee, JHEP 0701, (2007) 019 [arXiv:hep-ph/0609254].

[9] A. Karch, E. Katz, D. T. Son and M. A. Stephanov, Phys. Rev. D 74 (2006) 015005 [hep-ph/0602229].

[10] S. S. Gubser and A. Nellore, Phys. Rev. D 78 (2008) 086007 [arXiv:0804.0434 [hep-th]].

[11] O. DeWolfe and C. Rosen, JHEP 0907 (2009) 022 [arXiv:0903.1458 [hep-th]].

[12] H. Liu, K. Rajagopal and U. A. Wiedemann, Phys. Rev. Lett. 98 (2007) 182301 [arXiv:hep-ph/0607062].

[13] H. Liu, K. Rajagopal and Y. Shi, JHEP 0808, (2008) 048 [arXiv:0803.3214 [hep-ph]].

[14] D. Bak, A. Karch and L. G. Yaffe, JHEP 0708 (2007) 049 [arXiv:0705.0994 [hep-th]].

[15] C. P. Herzog, A. Karch, P. Kovtun, C. Kozcaz and L. G. Yaffe, JHEP 0607 (2006) 013 [arXiv:hep-th/0605158].

[16] S. S. Gubser, Phys. Rev. D 74 (2006) 126005 [arXiv:hep-th/0605182].

[17] E. Nakano, S. Teraguchi and W. Y. Wen, Phys. Rev. D 75 (2007) 085016 [arXiv:hep-ph/0608274].

[18] O. Kaczmarek, F. Karsch, F. Zantow and P. Petreczky, Phys. Rev. D 70 (2004) 074505 [Erratum-ibid. D 72 (2005) 059903] [arXiv:hep-lat/0406036].

[19] C. Athanasiou, P. M. Chesler, H. Liu, D. Nickel and K. Rajagopal, Phys. Rev. D 81 (2010) 126001 [Erratum-ibid. D 84 (2011) 069901] [arXiv:1001.3880 [hep-th]].

[20] K. B. Fadafan, H. Liu, K. Rajagopal and U. A. Wiedemann, Eur. Phys. J. C 61 (2009) 553 [arXiv:0809.2869 [hep-ph]]. 